\title{
THE HYPOLIPIDEMIC ACTIVITY OF HETEROCYCLIC THIOSEMICARBAZONES, THIOUREAS AND THEIR METAL COMPLEXES IN SPRAGUE DAWLEY MALE RATS
}

\author{
Iris H. Hall, S.Y. Chen ${ }^{1}$, Betsy Jo Barnes ${ }^{1}$ and Douglas X. West ${ }^{2}$ \\ ${ }^{1}$ Division of Medicinal Chemistry and Natural Products, School of Pharmacy, \\ University of North Carolina, Chapel Hill, North Carolina 27599-7360, USA \\ ${ }^{2}$ Department of Chemistry, Illinois State University, Noemal, Illinois 61761, USA
}

\begin{abstract}
Heterocyclic thiosemicarbazones, thioureas and their copper, nickel, and cobalt complexes were shown to be potent hypolipidemic agents in male Sprague Dawley rats at $8 \mathrm{mg} / \mathrm{kg} /$ day, orally. These agents lowered the activity of rat hepatic rate limiting enzymes for the synthesis of cholesterol and triglycerides. The effects of these agnets on cytoplasmic ATP-dependent citrate lyase, acetyl CoA synthetase and HMG-CoA reductase activities were reduced by a magnitude to explain the reduction of serum cholesterol levels afforded by the compounds. The reduction of acetyl CoA carboxylase, $s n$-glycerol-3-phosphate synthetase and phosphotidylate phosphohydrolase activities caused by the derivatives is of sufficient magnitude to explain the observed reduction in serum triglycerides after administration of the agents.
\end{abstract}

\section{Introduction}

Metal complexes containing copper, iron, cobalt, chromium, calcium and sodium of amine-carboxyboranes have previously been shown to have potent hypolipidemic activity in rodents at the low doses of 2.5 to 8 $\mathrm{mg} / \mathrm{kg}$ lowering both serum cholesterol and serum triglyceride levels [1-3]. These agents modulated rat lipoproteins in a manner so that LDL-cholesterol levels were reduced whereas HDL-cholesterol levels were elevated [4], which should lead to an enhanced clearance of cholesterol from the body. These complexes suppressed LDL-binding to tissue high affinity receptor thus reducing cholesterol uptake into peripheral tissue. Yet the complexes accelerated HDL-affinity binding to hepatic receptor so that serum cholesterol could be taken up and processed to bile salts and excreted via the biliary system. These metal complexes of amine carboxyboranes also demonstrated antineoplastic/cytotoxicity and antiinflammatory activity $[5,6]$. The metal complexes of thiosemicarbazones have also been shown to have antineoplastic and antiinflammatory activity [7-9]. The present study involved an investigation of their potential as hypolipidemic agents in rodent.

\section{Methods}

Source of Compounds

All of the derivatives [Fig 1] have been synthesized and reported in the literature previously [7].

\section{Hypolipidemic in vivo screen.}

Sprague Dawley male rats $[\sim 400 \mathrm{~g}]$ were administered drugs prepared in $1 \%$ carboxymethylcellulose [CMC] by homogenization at $8 \mathrm{mg} / \mathrm{kg} /$ day orally for 16 days. On days 9 and 16 the rats were bled from the tail vein collecxted into capillary tubes which were centrifuged at $3000 \mathrm{x} \mathrm{g}$ for $3 \mathrm{~min}$. Total cholesterol was determined on the serum by the Libermann-Burchard method [10] determined at $620 \mathrm{~nm}$. Serum triglycerides were determined on day 16 using a commercial kit from Sigma Chemical, Co. which was read at $580 \mathrm{~nm}$. Clofibrate at $150 \mathrm{mg} / \mathrm{kg} /$ day, orally, gemfibrozil at $90 \mathrm{mg} / \mathrm{kg} /$ day, orally and lovastatin at 8 $\mathrm{mg} / \mathrm{kg} /$ day, orally were determined at their standard therapeutic dose.

\section{Rat Hepatic Enzyme Studies in vitro}

Enzyme assays were performed on Sprague Dawley male rats $[\sim 450 \mathrm{~g}]$ livers homogenized $[10 \%]$ in 0.025 $\mathrm{M}$ sucrose $+0.001 \mathrm{M}$ EDTA, $\mathrm{pH}$ 7.2. Literature methods [11] were used to examine the effects of the compounds at 25,50 and $100 \mu \mathrm{M}$ over $60 \mathrm{~min}$. ATP dependent citrate lyase [12], acetyl CoA synthetase [13], HMG-CoA reductase [14,15], acyl CoA cholesterol acyl transferase [16], cholesterol-7 $\alpha$-hydroxylase [17], acetyl CoA carboxylase [18], sn-glycerol-3-phosphate acyl transferase [19], phospha-

tidylate phosphohydrolase[20] and hepatic lipoprotein lipase [21]. 


\section{Statistic Analysis}

Data displayed in the tables are the means \pm standard deviations of the mean expressed as percentage of the control. $\mathrm{N}$ is the number of samples or animals per group. The Student's " $\mathrm{t}$ "-test was used to determine the probable level of significance (p) between test samples and control samples.<smiles>C/C(=N\NC(=S)NC1CCCCCC1[18F])c1ccccn1</smiles><smiles>C/C(=N\NC(=S)N1CCCCCC1)c1ccccn1</smiles>

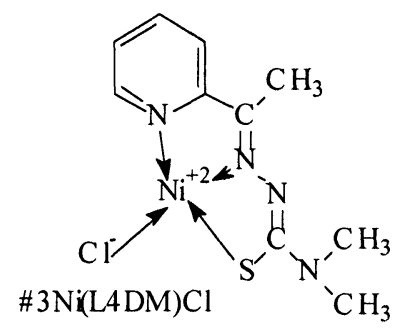

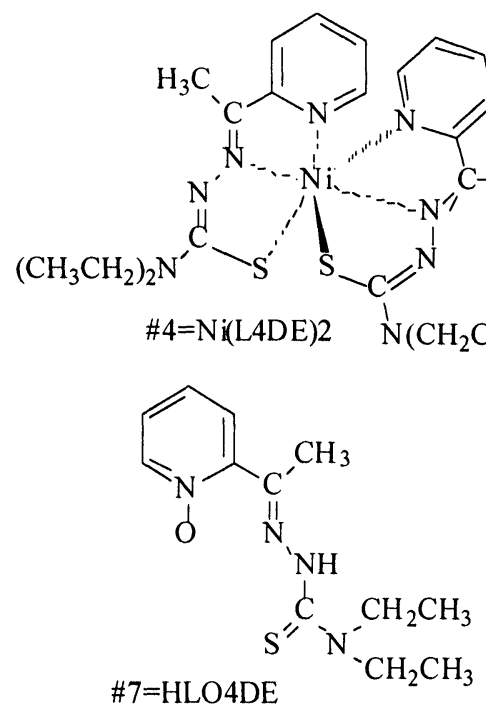

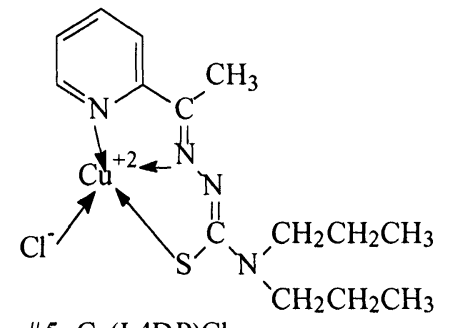

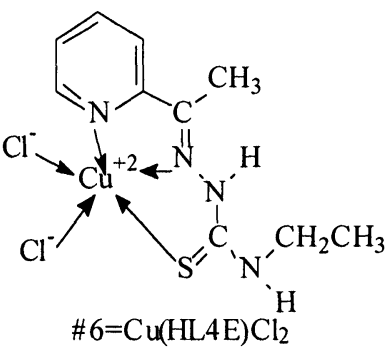
$\# 7=\mathrm{HLO} 4 \mathrm{DE}$

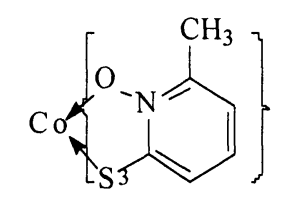<smiles>Cc1ccc(NC(=S)Nc2ccccc2C)nc1</smiles>

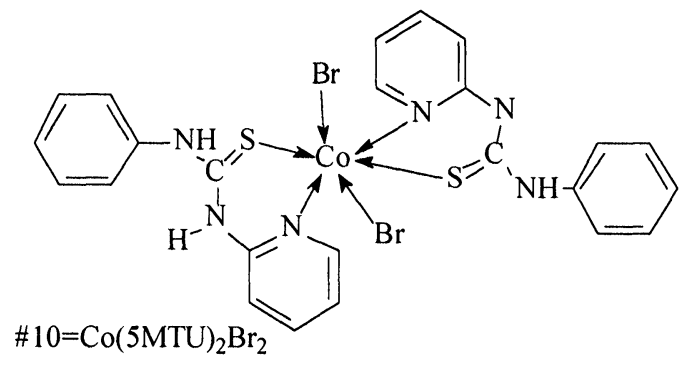

Fig. 1: Structures of Thiosemicarbazones and Their Metal Complexes

\section{Results}

All of the thiosemicarbazones demonstrated significant hypolipidemic activity in male Sprague Dawley rats at $8 \mathrm{mg} / \mathrm{kg} /$ day orally reducing both serum cholesterol and serum triglycerides by day 16 [Table 1]. These effects were superior to the standards clofibrate at $150 \mathrm{mg} / \mathrm{kg} / \mathrm{day}$, gemfibrozil at $90 \mathrm{mg} / \mathrm{kg} / \mathrm{day}$ and lovastatin at $8 \mathrm{mg} / \mathrm{kg} / \mathrm{day}$. On day 16 compounds $2,6,7$ and 8 caused greater than $40 \%$ reduction of serum cholesterol levels while compounds 3, 4, 5, 9 and 10 afforded greater than $32 \%$ reduction. Compounds 1 and 4 reduced serum triglycerides greater than $40 \%$ after 16 days and compounds 2, 3, 6, 8 and 10 caused at least $35 \%$ reduction of serum triglycerides. 
Table 1 Hypolipidemic Activity of Compounds 1-10 on Sprague Dawley Male Rats at $8 \mathrm{mg} / \mathrm{kg} / \mathrm{day}$ Orally

\begin{tabular}{|l|l|l|l|l|}
\hline Compound & \multicolumn{2}{|c|}{ Serum Cholesterol } & \multicolumn{2}{c|}{ Serum Triglycerides } \\
\hline$(\mathrm{N}=6)$ & Day 9 & Day 16 & Day 9 & Day 16 \\
\hline 1 & $81 \pm 5$ & $71 \pm 3^{*}$ & $76 \pm 3^{*}$ & $56 \pm 4^{*}$ \\
\hline 2 & $62 \pm 4^{*}$ & $59 \pm 4^{*}$ & $78 \pm 4^{*}$ & $64 \pm 4^{*}$ \\
\hline 3 & $93 \pm 5$ & $64 \pm 5^{*}$ & $72 \pm 2^{*}$ & $63 \pm 5^{*}$ \\
\hline 4 & $78 \pm 6^{*}$ & $61 \pm 5^{*}$ & $69 \pm 3^{*}$ & $59 \pm 3^{*}$ \\
\hline 5 & $71 \pm 5^{*}$ & $66 \pm 6^{*}$ & $82 \pm 5$ & $75 \pm 3^{*}$ \\
\hline 6 & $60 \pm 4^{*}$ & $55 \pm 4^{*}$ & $81 \pm 3$ & $61 \pm 5$ \\
\hline 7 & $52 \pm 4^{*}$ & $51 \pm 2^{*}$ & $84 \pm 5$ & $72 \pm 5^{*}$ \\
\hline 8 & $60 \pm 3^{*}$ & $57 \pm 5^{*}$ & $73 \pm 4^{*}$ & $65 \pm 4^{*}$ \\
\hline 9 & $82 \pm 4$ & $67 \pm 3^{*}$ & $79 \pm 2^{*}$ & $75 \pm 4^{*}$ \\
\hline 10 & $82 \pm 5$ & $62 \pm 4^{*}$ & $82 \pm 4$ & $62 \pm 3^{*}$ \\
\hline Clofibrate @ 150 mg/kg & $88 \pm 7$ & $86 \pm 2$ & $83 \pm 6$ & $74 \pm 7^{*}$ \\
\hline Gemfibrozil @ 90 mg/kg & $91 \pm 5$ & $82 \pm 7$ & $78 \pm 6^{*}$ & $62 \pm 5^{*}$ \\
\hline Lovastatin @ $\mathrm{mg} / \mathrm{kg}$ & $85 \pm 4$ & $78 \pm 5^{*}$ & $91 \pm 5$ & $86 \pm 7$ \\
\hline Control -1\% CMC & $100 \pm 5^{2}$ & $100 \pm 6^{\circ}$ & $100 \pm 7^{*}$ & $100 \pm 6^{\circ}$ \\
\hline
\end{tabular}

$a=73 \mathrm{mg} / \mathrm{dl} ; \mathrm{b}=75 \mathrm{mg} / \mathrm{dl} ; \mathrm{c}=111 \mathrm{mg} / \mathrm{dl} ; \mathrm{d}=112 \mathrm{mg} / \mathrm{dl}$

The activities of the regulatory enzymes involved in vitro hepatic de novo cholesterol and triglyceride syntheses and metabolism were significantly affected by the thiosemicarbazones and their metal complexes after $60 \mathrm{~min}$ incubation [Table 2-4]. Acetyl CoA synthetase activity was suppressed in a concentration manner causing greater than $50 \%$ reduction at $100 \mu \mathrm{M}$. The inhibition of ATP-dependent citrate lyase activity followed a similar pattern of inhibition by the agents but the magnitude of reduction was a little less than with acetyl CoA synthetase activity. The activity of the regulatory enzyme of cholesterol synthesis HMG-CoA reductase was reduced in a concentration dependent manner so that greater than $50 \%$ inhibition was achieved by compounds $3,4,6,7,8,9$ and 10. Compound 1 had the least effects on HMGCoA reductase activity after 1 hour and also had the least ability to lower serum cholesterol levels in vivo. The effects of the thiosemicarbazones on cholesterol-7 $\alpha$-hydroxylase activity were erratic, i.e compounds 4 , 9 and 10 had no effect or less than $20 \%$ reduction, compounds 1, 2, 3, 6 and 8 caused moderate reductions in activity of $24 \%$ to $41 \%$ and compound 7 afforded $62 \%$ reduction at $100 \mu \mathrm{M}$. Acyl-CoA cholesterol acyl transferase activity was reduced only moderately by compounds 1,7 and 8 by approximately $32 \%$.

Acetyl CoA carboxylase activity was also affected in an erratic manner by the compounds. Compounds $\mathbf{1 , 5}$, 6 and 7 resulted in $44 \%$ to $75 \%$ suppression after $60 \mathrm{~min}$ at $100 \mu \mathrm{M}$. Compounds 4,9 and 10 had marginal effects and compounds $\mathbf{3}$ and $\mathbf{8}$ had no effects on the activity. sn-Glycerol-3-phosphate acyl transferase activity was inhibited $48 \%$ to $70 \%$ by compounds $1,3,4,7,8$ and 10 and $25 \%$ to $36 \%$ by compounds 5 and 6 but compound 9 had no effect. Phosphatidylate phosphohydrolase activity was markedly reduced greater than $70 \%$ at $100 \mu \mathrm{M}$ with the exception of compound 9 which only caused $57 \%$ reduction. Hepatic lipoprotein lipase activity was essentially unaffected by compounds $3,5,7,8$ and 9 , and was moderately reduced by compounds 4,6 and 10. Compounds 1 and 2 caused reduction of hepatic lipoprotein lipase activity of $45 \%$ to $59 \%$ after $60 \mathrm{~min}$ incubation

\section{Discussion}

The observed reduction in the activities of the hepatic regulatory enzymes caused by the thiosemicarbazones and their metal complexes are of a magnitude to account for the reductions of serum cholesterol and triglyceride levels after 16 days in vivo. The reduction of acetyl CoA synthetase

and ATP dependent citrate lyase activities not only would reduce the synthesis of cholesterol in the cytoplasm of the cell but would also lower the levels of fatty acids and neutral lipids since acetyl-CoA is the common starting intermediate for all of the synthestic pathoways for these lipids. The fact that there was a positive correlation between the ability of the compounds to reduce the activity of HMG-CoA reductase and its ability to lower serum cholesterol levels indicates that this may be a key target of the thiosemicarbazones and their metal complexes.

Reduction of cholesterol-7 $\alpha$-hydroxylase activity by a few compounds would lead to less cholesterol being converted to bile acids for biliary excretion. For the few compounds that inhibited acyl-CoA cholesterol acyl transferase activity less cholesterol esters would be stored in the liver. If the analogous reaction occurred in aorta foam cells then reducing cholesterol esters would reduce the size of the plaque.

Table 2: The Effects of Thiosemicarbazones on Sprague Dawley Liver Enzyme Activities 


\begin{tabular}{|c|c|c|c|c|c|c|c|}
\hline Assay & Control & \multicolumn{3}{|c|}{ Compound 1} & \multicolumn{3}{|c|}{ Compound 2} \\
\hline$(\mathrm{N}=6)$ & & $25 \mu \mathrm{M}$ & $50 \mu \mathrm{M}$ & $100 \mu \mathrm{M}$ & $25 \mu \mathrm{M}$ & $50 \mu \mathrm{M}$ & $100 \mu \mathrm{M}$ \\
\hline Acetyl CoA Synthetase ${ }^{a}$ & $100 \pm 5$ & $69 \pm 5^{*}$ & $47 \pm 4^{*}$ & $20 \pm 2 *$ & $99 \pm 6$ & $63 \pm 3^{*}$ & $47 \pm 4^{*}$ \\
\hline ATP dependent Citrate Lyase ${ }^{\circ}$ & $100 \pm 4$ & $65 \pm 4^{*}$ & $51 \pm 4^{*}$ & $38 \pm 3^{*}$ & $48 \pm 4$ & $36 \pm 4^{*}$ & $30 \pm 3^{*}$ \\
\hline HMG-CoA reductase ${ }^{c}$ & $100 \pm 6$ & $87 \pm 5$ & $87 \pm 6$ & $80 \pm 4^{*}$ & $80 \pm 4^{*}$ & $61 \pm 4^{*}$ & $57 \pm 5^{*}$ \\
\hline Cholesterol-7 $\alpha$ hydroxylase ${ }^{\alpha}$ & $100 \pm 7$ & $104 \pm 6$ & $89 \pm 5$ & $79 \pm 5^{*}$ & $96 \pm 6$ & $90 \pm 5$ & $75 \pm 5^{*}$ \\
\hline Acyl-CoA Cholesterol Acyl Transferase & $100 \pm 5$ & $107 \pm 6$ & $84 \pm 5$ & $65 \pm 5$ & $116 \pm 6$ & $82 \pm 6$ & $80 \pm 4^{*}$ \\
\hline Acetyl CoA carboxylase & $100 \pm 4$ & $61 \pm 5^{*}$ & $51 \pm 4^{*}$ & $37 \pm 4^{*}$ & $68 \pm 4^{*}$ & $63 \pm 4^{*}$ & $56 \pm 3^{*}$ \\
\hline sn-Glycerol-3-Phosphate ${ }^{3}$ Acyl Transferase ${ }^{n}$ & $100 \pm 5$ & $86 \pm 5$ & $64 \pm 5^{*}$ & $40 \pm 3^{*}$ & $61 \pm 3^{*}$ & $57 \pm 5^{*}$ & $52 \pm 4^{*}$ \\
\hline Phosphatidylate Phosphohydrolase & $100 \pm 6$ & $79 \pm 4^{*}$ & $37 \pm 4^{*}$ & $17 \pm 3^{*}$ & $85 \pm 6$ & $39 \pm 3^{*}$ & $19 \pm 2^{*}$ \\
\hline Hepatic Lipoprotein Lipase & $100 \pm 5$ & $67 \pm 3^{*}$ & $61 \pm 4^{*}$ & $55 \pm 4^{*}$ & $51 \pm 4^{*}$ & $45 \pm 4^{*}$ & $41 \pm 3^{*}$ \\
\hline
\end{tabular}

\begin{tabular}{|l|l|l|l|l|l|l|l|}
\hline Assay & Control & \multicolumn{3}{c|}{ Compound 7 } & \multicolumn{3}{c|}{ Compound 9 } \\
\hline$(\mathrm{N}=6)$ & & $25 \mu \mathrm{M}$ & $50 \mu \mathrm{M}$ & $100 \mu \mathrm{M}$ & $25 \mu \mathrm{M}$ & $50 \mu \mathrm{M}$ & $100 \mu \mathrm{M}$ \\
\hline Acetyl CoA Synthetase & $100 \pm 5$ & $128 \pm 8$ & $52 \pm 5^{*}$ & $38 \pm 4^{*}$ & $110 \pm 7$ & $50 \pm 5^{*}$ & $28 \pm 4^{*}$ \\
\hline ATP dependent Citrate Lyase & $100 \pm 4$ & $52 \pm 5^{*}$ & $39 \pm 5^{*}$ & $35 \pm 4^{*}$ & $97 \pm 6$ & $94 \pm 5$ & $67 \pm 4^{*}$ \\
\hline HMG-CoA reductase & $100 \pm 6$ & $57 \pm 4^{*}$ & $53 \pm 5^{*}$ & $48 \pm 3^{*}$ & $48 \pm 4^{*}$ & $43 \pm 3^{*}$ & $43 \pm 3^{*}$ \\
\hline Cholesterol-7 $\alpha$ hydroxylase & $100 \pm 7$ & $74 \pm 3^{*}$ & $52 \pm 4^{*}$ & $38 \pm 4^{*}$ & $132 \pm 6^{*}$ & $115 \pm 5$ & $97 \pm 6$ \\
\hline Acyl-CoA Cholesterol Acyl Transferase & $100 \pm 5$ & $99 \pm 7$ & $82 \pm 5$ & $67 \pm 4^{*}$ & $146 \pm 8^{*}$ & $123 \pm 6^{*}$ & $97 \pm 5$ \\
\hline Acetyl CoA carboxylase & $100 \pm 4$ & $54 \pm 5^{*}$ & $44 \pm 4^{*}$ & $40 \pm 2^{*}$ & $87 \pm 5^{*}$ & $72 \pm 4^{*}$ & $63 \pm 3^{*}$ \\
\hline Sn-Glycerol-3-Phosphate Acyl Transferase & $100 \pm 5$ & $108 \pm 6$ & $80 \pm 5^{*}$ & $30 \pm 3^{*}$ & $131 \pm 5^{*}$ & $100 \pm 6$ & $97 \pm 5$ \\
\hline Phosphatidylate Phosphohydrolase & $100 \pm 6$ & $58 \pm 5^{*}$ & $28 \pm 3^{*}$ & $19 \pm 2^{*}$ & $129 \pm 6^{*}$ & $60 \pm 5^{*}$ & $43 \pm 4^{*}$ \\
\hline Hepatic Lipoprotein Lipase & $100 \pm 5$ & $112 \pm 5$ & $84 \pm 5$ & $83 \pm 4$ & $98 \pm 6$ & $95 \pm 5$ & $89 \pm 6$ \\
\hline
\end{tabular}

$\mathrm{a}=10.1 \mathrm{mg}$ acetyl CoA formed $/ \mathrm{g}$ wet tissue; $\mathrm{b}=9.2 \mathrm{mg}$ citrate hydrolyzed/g wet tissue; $\mathrm{c}=103,020$ $\mathrm{dpm} / \mathrm{g}$ wet tissue; $\mathrm{d}=289,450 \mathrm{dpm} / \mathrm{g}$ wet tissue; $\mathrm{e}=86,640 \mathrm{dpm} / \mathrm{g}$ wet tissue; $\mathrm{f}=43021 \mathrm{dpm} / \mathrm{g}$ wet tissue; $\mathrm{g}=87,620 \mathrm{dpm} / \mathrm{g}$ wet tissue; $\mathrm{h}=11 \mu \mathrm{g}$ Pi released $/ \mathrm{g}$ wet tissue; $\mathrm{i}=234,675 \mathrm{dpm} / \mathrm{g}$ wet tissue.

Table 3 The Effects of Nickel Complexes of Thiosemicarbazones On Sprague Dawley Liver Enzyme Activities

\begin{tabular}{|c|c|c|c|c|c|c|c|}
\hline Assay & Control & \multicolumn{3}{|c|}{ Compound 3} & \multicolumn{3}{|c|}{ Compound 4} \\
\hline$(\mathrm{N}=6)$ & & $25 \mu \mathrm{M}$ & $50 \mu \mathrm{M}$ & $100 \mu \mathrm{M}$ & $25 \mu \mathrm{M}$ & $50 \mu \mathrm{M}$ & $100 \mu \mathrm{M}$ \\
\hline Acetyl CoA Synthetase ${ }^{a}$ & $100 \pm 5$ & $46 \pm 4^{*}$ & $31 \pm 4^{*}$ & $19 \pm 3^{*}$ & $98 \pm 5$ & $48 \pm 4^{*}$ & $10 \pm 2^{*}$ \\
\hline ATP dependent Citrate Lyase & $100 \pm 4$ & $69 \pm 5^{*}$ & $49 \pm 3^{*}$ & $39 \pm 2^{*}$ & $80 \pm 4^{*}$ & $58 \pm 3^{*}$ & $47 \pm 3^{*}$ \\
\hline HMG-CoA reductase ${ }^{c}$ & $100 \pm 6$ & $65 \pm 4^{*}$ & $53 \pm 3^{*}$ & $42 \pm 4^{*}$ & $76 \pm 4^{*}$ & $48 \pm 3^{*}$ & $48 \pm 4^{*}$ \\
\hline Cholesterol-7 $\alpha$ hydroxylase $^{\alpha}$ & $100 \pm 7$ & $\overline{93 \pm 5}$ & $76 \pm 3^{*}$ & $62 \pm 3^{*}$ & $112 \pm 6$ & $97 \pm 5$ & $84 \pm 4$ \\
\hline Acyl-CoA Cholesterol Acyl Transferase ${ }^{e}$ & $100 \pm 5$ & $116 \pm 6$ & $88 \pm 5$ & $77 \pm 5$ & $88 \pm 5$ & $87 \pm 5$ & $82 \pm 4$ \\
\hline Acetyl CoA carboxylase & $100 \pm 4$ & $115 \pm 6$ & $96 \pm 5$ & $87 \pm 6$ & $102 \pm 5$ & $92 \pm 6$ & $72 \pm 4 *$ \\
\hline sn-Glycerol-3-Phosphate ${ }^{3}$ Acyl Transferase & $100 \pm 5$ & $56 \pm 4^{*}$ & $54 \pm 3^{*}$ & $51 \pm 3^{*}$ & $67 \pm 3^{*}$ & $60 \pm 6$ & $46 \pm 3^{*}$ \\
\hline Phosphatidylate Phosphohydrolase & $100 \pm 6$ & $70 \pm 3^{*}$ & $29 \pm 3 *$ & $23 \pm 3$ & $100 \pm 4$ & $46 \pm 4^{*}$ & $21 \pm 3 *$ \\
\hline Hepatic Lipoprotein Lipase & $100 \pm 5$ & $129 \pm 7$ & $90 \pm 6$ & $90 \pm 6$ & $104 \pm 5$ & $97 \pm 6$ & $73 \pm 5^{*}$ \\
\hline
\end{tabular}

\begin{tabular}{|l|l|l|l|l|l|l|l|}
\hline Assay & Control & \multicolumn{3}{|c|}{ Compound 5 } & \multicolumn{3}{c|}{ Compound 6 } \\
\hline$(\mathrm{N}=6)$ & & $25 \mu \mathrm{M}$ & $50 \mu \mathrm{M}$ & $100 \mu \mathrm{M}$ & $25 \mu \mathrm{M}$ & $50 \mu \mathrm{M}$ & $100 \mu \mathrm{M}$ \\
\hline Acetyl CoA Synthetase & $100 \pm 5$ & $121 \pm 5$ & $67 \pm 4^{*}$ & $42 \pm 4^{*}$ & $72 \pm 4^{*}$ & $48 \pm 4^{*}$ & $32 \pm 3^{*}$ \\
\hline ATP dependent Citrate Lyase & $100 \pm 4$ & $93 \pm 6$ & $65 \pm 5^{*}$ & $53 \pm 5^{*}$ & $80 \pm 6$ & $54 \pm 3^{*}$ & $41 \pm 2^{*}$ \\
\hline HMG-CoA reductase & $100 \pm 6$ & $67 \pm 5^{*}$ & $57 \pm 5^{*}$ & $56 \pm 4^{*}$ & $66 \pm 4^{*}$ & $42 \pm 4^{*}$ & $40 \pm 3^{*}$ \\
\hline Cholesterol-7 $\alpha$ hydroxylase & $100 \pm 7$ & $107 \pm 6$ & $88 \pm 6$ & $76 \pm 5^{*}$ & $89 \pm 6$ & $81 \pm 4$ & $59 \pm 4^{*}$ \\
\hline Acyl-CoA Cholesterol Acyl Transferase & $100 \pm 5$ & $89 \pm 7$ & $87 \pm 6$ & $83 \pm 5$ & $122 \pm 5$ & $99 \pm 6$ & $86 \pm 5$ \\
\hline Acetyl CoA carboxylase & $100 \pm 4$ & $45 \pm 4^{*}$ & $35 \pm 3^{*}$ & $25 \pm 4$ & $49 \pm 4^{*}$ & $42 \pm 4^{*}$ & $42 \pm 3^{*}$ \\
\hline sn-Glycerol-3-Phosphate Acyl Transferase & $100 \pm 5$ & $124 \pm 5$ & $107 \pm 6$ & $75 \pm 4^{*}$ & $116 \pm 6$ & $66 \pm 5^{*}$ & $64 \pm 3^{*}$ \\
\hline Phosphatidylate Phosphohydrolase & $100 \pm 6$ & $44 \pm 3^{*}$ & $25 \pm 4^{*}$ & $19 \pm 3^{*}$ & $48 \pm 3^{*}$ & $26 \pm 3^{*}$ & $13 \pm 2^{*}$ \\
\hline Hepatic Lipoprotein Lipase & $100 \pm 5$ & $149 \pm 7^{*}$ & $140 \pm 8^{*}$ & $95 \pm 6$ & $89 \pm 4$ & $83 \pm 5$ & $73 \pm 4^{*}$ \\
\hline
\end{tabular}

The reduction of acetyl CoA carboxylase activity by the thiosemicarbazones and their metal complexes would lead to less synthesis of fatty acids and the suppression of $s n$-glycerol-3-phosphate acyl transferase activity would lead to less neutral lipid synthesis. The ability of the compounds to lower phosphatidylate phosphohydrolase activity would block the removal of the phosphorus moiety from phospholipids so that triglyceride could be formed. This again appears to be major target of the agents in lowering serum triglyceride levels. 
Interestingly there did not appear to be any differences in the effects of thiosemicarbazones compared to their metal complexes on either the serum lipid levels or the enzyme activities. Nor did any one type of metal complex appear to be better in reducing these parameters compared to another. The amine carboxyboranes and their metal complexes also demonstrated a pattern of having multiple target enzymes within de novo lipid synthesis [1-3]. These derivatives had more effect on acyl-CoA cholesterol acyl transferase activity than the thiosemicarbazones and its metal complexes while the thiosemicarbazones and their metal complexes had more effects on HMG-CoA reductase activity than the amine carboxyboranes and their metal complexes. Similar effects were observed on the other enzyme activities examined by both groups of compounds as well as the magnitude of serum cholesterol and triglyceride reduction in rodents.

Table 4 The Effects of Cobalt Complexes of Thiosemicarbazones On Sprague Dawley Liver Enzyme Activities

\begin{tabular}{|c|c|c|c|c|c|c|c|}
\hline Assay & Control & \multicolumn{3}{|c|}{ Compound $\mathbf{8}$} & \multicolumn{3}{|c|}{ Compound 10} \\
\hline$(\mathrm{N}=6)$ & & $25 \mu \mathrm{M}$ & $50 \mu \mathrm{M}$ & $100 \mu \mathrm{M}$ & $25 \mu \mathrm{M}$ & $50 \mu \mathrm{M}$ & $100 \mu \mathrm{M}$ \\
\hline Acetyl CoA Synthetase & $100 \pm 5$ & $83 \pm 5^{*}$ & $57 \pm 4^{*}$ & $36 \pm 3^{*}$ & $53 \pm 4^{*}$ & $33 \pm 3^{*}$ & $20 \pm 3^{*}$ \\
\hline ATP dependent Citrate Lya & $100 \pm 4$ & $63 \pm 4^{*}$ & $39 \pm 3$ & $35 \pm 3^{*}$ & $111 \pm 5$ & $68 \pm 4^{*}$ & $43 \pm 3^{*}$ \\
\hline HMG-CoA reductase $^{c}$ & $100 \pm 6$ & $54 \pm 5^{*}$ & $48 \pm 3^{*}$ & $32 \pm 4^{*}$ & $56 \pm 5^{*}$ & $46 \pm 4^{*}$ & $45 \pm 4^{*}$ \\
\hline Cholesterol-7 $\alpha$ hydroxylase ${ }^{\alpha}$ & $100 \pm 7$ & $79 \pm 6^{*}$ & $71 \pm 4^{*}$ & $57 \pm 4^{*}$ & $118 \pm 6$ & $108 \pm 5$ & $93 \pm 5$ \\
\hline sterol Acyl Tra & $100 \pm 5$ & $101 \pm 5$ & $74 \pm 5^{*}$ & $61 \pm 4^{*}$ & $110 \pm 5$ & $84 \pm 5$ & $77 \pm 3^{*}$ \\
\hline Acetyl Co & $100 \pm 4$ & $70 \pm 4^{*}$ & $96 \pm 5$ & $87 \pm 6$ & $102 \pm 5$ & $92 \pm 6$ & $72 \pm 4^{*}$ \\
\hline sn-Glycerol-3-Phosphate ${ }^{3}$ Acyl Transferase ${ }^{n}$ & $100 \pm 5$ & $56 \pm 4^{*}$ & $54 \pm 3^{*}$ & $51 \pm 3^{*}$ & $67 \pm 3^{*}$ & $60 \pm 4^{*}$ & $46 \pm 3^{*}$ \\
\hline Phosphatidylate Phosphohydrolase & $100 \pm 6$ & $70 \pm 5^{*}$ & $29 \pm 3^{*}$ & $23 \pm 3^{*}$ & $100 \pm 4$ & $46 \pm 4^{*}$ & $21 \pm 3^{*}$ \\
\hline Hepatic Lipoprotein Lipas & $100 \pm 5$ & $129 \pm 7^{*}$ & $90 \pm 5$ & $90 \pm 6$ & $104 \pm 5$ & $97 \pm 6$ & $73 \pm 5^{*}$ \\
\hline
\end{tabular}

\section{References}

1. I.H. Hall, B.F. Spielvogel, A. Sood, K.W. Morse, V. M. Noewood, III, O.T. Wong, Metal Based Drugs 1, 329-336, 1994.

2. I.H. Hall, W.L. Williams, Jr.,C.J. Gilbert, A.T. McPhail, and B.F. Spielvogel, J. Pharm. Sci. 73, 973-977, 1984.

3. I H. Hall, B.S. Burnham, S.Y. Chen, A. Sood, B.F. Spielvogel, and K.W. Morse, Metal Based Drugs 2, 1-12, 1995.

4. I. H. Hall, B.F. Spielvogel, T.S. Griffin, E.L. Docks, R.J. Brotherton, Res. Commun. Chem. Path. \& Pharmacol 65, 297-314, 1989.

5 I.H. Hall, B.F. Spielvogel, A. Sood, F. Ahmed, S. Jafri, J. Pharm. Sci. 76, 359-365, 1987.

6. A. Sood, C.K. Sood, B.F. Spielvogel, I.H. Hall, I.H., Eur. J. Med. Chem. 25, 301-308, 1990.

7. D.X. West, A.E. Liberta, K.G. Rajendran, and I.H. Hall, Anti-Cancer Drugs 4, 241-249, 1993.

8. I.H. Hall, K.G. Raijendra, D.X. West, A.E. Liberta, Anti-Cancer Drugs 4, 231-240, 1993.

9. I.H. Hall, S.Y. Chen, K.G. Rajendran, and D.X. West, Appl. Organomet Chem.10, 485-493,1996.

10. A.T. Ness, J.V. Pastewka, A.C. Paecock, Clin. Chim. Acta 10, 229-237, 1964.

11. I.H Hall, O.T. Wong, R. Simlot, D.J. Reynolds, Pharm. Res. 9, 1324-1329, 1992.

12. M. Hoffman, L. Weiss, O.H. Wieland, Anal. Biochem. 84, 441-448, 1978.

13. A.G. Goodridge, J. Biol. Chem. 248, 4318-4327, 1973.

14. G.T. Haven, J.R. Kremian, T.T. Nguyen, Res. Commun, Chem. Pathol. Pharmacol. 6, 253-261, 1973.

15. F. Wada, K. Hirata, Y. Sakameto, J. Biochem [Tokyo], 65, 171-175, 1989.

16. S. Balsasbramaniam, K.A. Mitropoulos, S. Venkatean, Eur. J. Biochem. 90, 377-383, 1978.

17. S. Shefer, S. Hauser, E.H. Mosbach, J. Lipid Res. 19, 467-477, 1978.

18. M.D. Greenspan, J.M. Lowenstein, J. Biol. Chem. 243, 6273-6280, 1968.

19. R.G. Lamb, S.D. Wyrick, C. Piantadosi, Artherscol. 27, 147-154, 1977.

20. R.D. Mavis, N. Jacob, J.N. Finkelstein, B.P. Hall, J. Lipid Res. 19, 467-477, 1978.

21. A. Chait P.h. Iverius, J.D. Brunzell, J. Clin. Invest. 69, 490-493, 1982.

Received: March 22, 1999 - Accepted: April 23, 1999 Received in revised camera-ready format: April 24, 1999 